\title{
ENTROPY AND THE HEAT EQUATION
}

\author{
BY \\ W. A. DAY \\ Hertford College, Oxford, England
}

1. Introduction. Until recent times there was little overlap between the disciplines of heat conduction and thermodynamics. (For extensive comment on this state of affairs, see Truesdell [1, pp. 47-137].) In two earlier papers [2,3] I proved certain theorems about the differential equations of heat conduction whose interest lies in the fact that they draw upon ideas imported from thermodynamics and involve the notion of entropy. The present paper examines how some of the conclusions of [3], which is concerned exclusively with steady-state heat conduction, are modified when the heat conduction is time-dependent.

Let us consider a rigid heat conductor that is homogeneous and isotropic and that occupies a domain $\Omega$ of euclidean three-space $\mathbf{R}^{3}$. In Sec. 2 the domain $\Omega$ is required to be the whole of $\mathbf{R}^{3}$, while in Sec. 3 it is a half-space. For the sake of simplicity we suppose the thermal conductivity and the heat capacity of unit volume to be equal to unity. The temperature $u(x, t)$, at the point $x=\left(x_{1}, x_{2}, x_{3}\right) \in \Omega$ and at time $t$, satisfies the inhomogeneous heat equation

$$
u_{t}=\Delta u+f(x) \text {, }
$$

where

$$
\Delta=\frac{\partial^{2}}{\partial x_{1}^{2}}+\frac{\partial^{2}}{\partial x_{2}^{2}}+\frac{\partial^{2}}{\partial x_{3}^{2}}
$$

is the Laplace operator and $f(x)$ is the heat supply to unit volume in unit time.

It should be noted that we presume the heat supply $f$ to be independent of time. We presume, in addition, that we are given a nonempty, bounded domain $D \subset \Omega$, and we require that $f$ meet the following conditions:

$$
\begin{gathered}
f(x) \geq 0 \text { for all } x \in \Omega, \\
\operatorname{supp} f \subset D, \\
f \in C^{\alpha}, \text { with a Hölder exponent } \alpha \text { in } 0<\alpha<1 .
\end{gathered}
$$

These conditions place no restriction upon the size of the integral

$$
\int_{\Omega} f d x\left(=\int_{D} f d x\right)
$$

Received December 26, 1991.

1991 Mathematics Subject Classification. Primary 35K05, 80A10. 
which can be made arbitrarily large. We shall assume that we can vary $f$ at will, subject only to the requirements (1.2), (1.3), and (1.4). The collection, $\mathscr{C}$ say, of all such heat supplies $f$ is a cone, that is,

$$
\lambda f+\mu g \in \mathscr{C} \text { whenever } f, g \in \mathscr{C} \text { and } \lambda, \mu \geq 0 .
$$

We shall write

$$
\begin{aligned}
& v=\int_{D} d x, \\
& \delta=\sup \{|x-y|: x, y \in D\}, \\
& \rho=\sup \{|x|: x \in D\},
\end{aligned}
$$

respectively, for the volume of $D$, the diameter of $D$, and the maximum distance of points of $D$ from the origin.

If $u$ is positive, as will be the case if the temperature is measured on an absolute scale, we may divide Eq. (1.1) through by $u$ and obtain the equation

$$
\frac{u_{t}}{u}+\operatorname{div}\left(\frac{q}{u}\right)=\frac{|q|^{2}}{u^{2}}+\frac{f}{u},
$$

in which

$$
q=-\operatorname{grad} u
$$

is the heat-flux vector. Thus if $P \subset \Omega$ is a bounded subdomain, with smooth boundary $\partial P$, then on integrating both sides of Eq. (1.9) over $P$ and appealing to the divergence theorem, we arrive at the identity

$$
\int_{P} \frac{u_{t}}{u} d x+\int_{\partial P} \frac{\nu \cdot q}{u} d S=\int_{P} \frac{|q|^{2}}{u^{2}} d x+\int_{P} \frac{f}{u} d x
$$

in which $\nu$ is the outward unit normal to $\partial P, d S$ is the element of surface area on $\partial P$, and $d x$ is the element of volume.

The terms that occur in the identity (1.10) have the following interpretations:

$\int_{P} \frac{u_{t}}{u} d x=\frac{d}{d t} \int_{P} \log u d x=$ rate of increase of entropy in $P$,

$\int_{\partial P} \frac{\nu \cdot q}{u} d S=$ flux of entropy out of $P$ due to heat conduction across $\partial P$,

$\int_{P} \frac{|q|^{2}}{u^{2}} d x=$ rate of generation of entropy within $P$ due to internal heat conduction.

$\int_{P} \frac{f}{u} d x=$ flux of entropy into $P$ due to the heat supply $f$.

With Eq. (1.10) in mind we set

$$
\begin{aligned}
& E(f, t)=\int \frac{u_{t}}{u} d x, \\
& F(f, t)=\int \frac{f}{u} d x, \\
& G(f, t)=\int \frac{|q|^{2}}{u^{2}} d x, \\
& H(f, t)=G(f, t)+F(f, t)-E(f, t),
\end{aligned}
$$


the integrals being extended over all of $\Omega$, and we make the following interpretations: $E(f, t)=$ rate of increase of entropy at time $t$, $F(f, t)=$ inward flux of entropy at time $t$ due to the heat supply, $G(f, t)=$ rate of generation of entropy at time $t$ due to internal heat conduction, $H(f, t)=$ outward flux of entropy at time $t$ due to heat conduction at infinity or across the boundary of $\Omega$.

The interpretation of $H(f, t)$ is made with the gloss that if $\Omega=\mathbf{R}^{3}$, then $H(f, t)$ is the outward flux of entropy at infinity but that if $\Omega$ is the half-space $x_{1}>0$, then $H(f, t)$ is the sum of the outward flux of entropy at infinity and the outward flux of entropy across the plane $x_{1}=0$.

Our objective is to study how large $E, F, G$, and $H$ can be made, for a given $t$, by varying the heat supply within the collection $\mathscr{C}$.

2. Behaviour of $E, F, G, H$ when $\Omega=\mathbf{R}^{3}$. We turn first to the case in which the conductor occupies the whole of space. The results of this section are counterparts, in the time-dependent case, to the results of Sec. 2 of [3].

Let $u_{0}$ be a positive constant. We shall require that the temperature satisfy the differential equation (1.1), together with the initial condition

$$
u(x, 0)=u_{0} \text { for all } x \in \mathbf{R}^{3}
$$

and the condition

$$
u(x, t) \rightarrow u_{0} \text { as }|x| \rightarrow \infty \text { for } t>0 .
$$

A standard argument assures us that the Cauchy problem consisting of Eqs. (1.1), (2.1), and (2.2) has at most one solution and, indeed, that we can construct it explicitly as

$$
u(x, t)= \begin{cases}u_{0}+\frac{1}{4 \pi} \int_{D} \frac{1}{|x-y|} \operatorname{erfc}\left(\frac{|x-y|}{2 t^{1 / 2}}\right) f(y) d y & \text { for } t>0, \\ u_{0} & \text { for } t=0 .\end{cases}
$$

In fact, we can prove that if $f \in \mathscr{C}$, then Eq. (2.3) defines a function $u$ that is continuous for $t \geq 0$ and that satisfies the initial condition (2.1) and the condition (2.2) and that also satisfies the differential equation (1.1) for $t>0$.

In order to verify these claims we note first that the continuity of $u$ for $t>0$ is an immediate consequence of familiar results from integration theory. In order to check that $u$ is actually continuous when $t=0$ we note that, as an integration by parts shows,

$$
\begin{aligned}
\operatorname{erfc}(\mu) & =\frac{2}{\pi^{1 / 2}} \int_{\mu}^{\infty} \exp \left(-\lambda^{2}\right) d \lambda \\
& =\frac{1}{\pi^{1 / 2} \mu} \exp \left(-\mu^{2}\right)-\frac{1}{\pi^{1 / 2}} \int_{\mu}^{\infty} \frac{1}{\lambda^{2}} \exp \left(-\lambda^{2}\right) d \lambda .
\end{aligned}
$$

Thus, the complementary error function satisfies the inequalities

$$
0<\operatorname{erfc}(\mu)<\frac{1}{\pi^{1 / 2} \mu} \exp \left(-\mu^{2}\right)<\frac{1}{\pi^{1 / 2} \mu} \text { for } \mu>0
$$


and, on combining these with Eq. (2.3), we see that

$$
0<u(x, t)-u_{0}<\frac{M t^{1 / 2}}{2 \pi^{3 / 2}} \int_{D} \frac{1}{|x-y|^{2}} d y \quad \text { for } t>0,
$$

where $M$ is an upper bound on $f$. A known estimate for improper integrals $[4$, p. 172] tells us that

$$
\int_{D} \frac{1}{|x-y|^{2}} d y \leq\left(48 \pi^{2} v\right)^{1 / 3} \text { for all } x \in \mathbf{R}^{3},
$$

and so

$$
0<u(x, t)-u_{0}<\pi^{-5 / 6} M(6 v)^{1 / 3} t^{1 / 2} .
$$

This last inequality confirms the continuity of $u$ at the initial time $t=0$. It is, of course, clear from Eq. (2.3) that the initial condition (2.1) is satisfied.

To check that condition (2.2) is satisfied we need only note that $0<\operatorname{erfc}(\mu) \leq 1$ and, therefore, that if $\rho$ is the maximum distance defined by Eq. (1.8), then

$$
0<u(x, t)-u_{0} \leq \frac{1}{4 \pi(|x|-\rho)} \int_{D} f(y) d y \quad \text { for }|x|>\rho .
$$

Finally, we rewrite $u$ in the form

$$
u(x, t)=u_{0}+v(x)-w(x, t),
$$

in which $v(x)$ is the Newtonian potential

$$
\frac{1}{4 \pi} \int_{D} \frac{1}{|x-y|} f(y) d y
$$

and

$$
w(x, t)=\frac{1}{4 \pi} \int_{D} \frac{1}{|x-y|} \operatorname{erf}\left(\frac{|x-y|}{2 t^{1 / 2}}\right) f(y) d y .
$$

Since $f$ is Hölder continuous, $v$ satisfies Poisson's equation $\Delta v=-f$, while, as differentiation under the integral sign reveals, $w$ is a solution of the heat equation $w_{t}=\Delta w$ for $t>0$. We conclude that $u$ must be a solution of the differential equation (1.1) for $t>0$.

We turn now to showing that $E, F, G$, and $H$ are well defined by Eqs. (1.11), (1.12), (1.13), and (1.14), respectively, the integrals being extended over all of $\mathbf{R}^{3}$. We shall show that for each $f \in \mathscr{C}$ and each $t>0, E, F$, and $G$ exist as Lebesgue integrals and are nonnegative; moreover,

$$
E=G+F
$$

and, hence,

$$
H=0 .
$$

These conclusions should be contrasted with those of Lemma 1 of [3], which deals with the corresponding steady-state case in which the temperature $u(x)$ is the solution of the problem

$$
\left\{\begin{aligned}
\Delta u+f(x) & =0 & & \text { for all } x \\
u(x) & \rightarrow u_{0} & & \text { as }|x| \rightarrow \infty .
\end{aligned}\right.
$$


In those circumstances $E$ is of necessity equal to zero but $H$ has the value

$$
\frac{1}{u_{0}} \int_{D} f d x
$$

In rough terms we might say that, in the time-dependent case, no finite time $t$, however large, is sufficient for there to be a positive outward flux of entropy due to heat conduction at infinity.

In order to verify our assertions we start by differentiating both sides of Eq. (2.3) with respect to $t$ and find that

$$
u_{t}=\frac{1}{(4 \pi t)^{3 / 2}} \int_{D} \exp \left(-\frac{|x-y|^{2}}{4 t}\right) f(y) d y>0 .
$$

Thus, if $\rho$ is the maximum distance defined by Eq. (1.8), we have

$$
0<u_{t} \leq \frac{1}{(4 \pi t)^{3 / 2}} \exp \left(-\frac{(|x|-\rho)^{2}}{4 t}\right) \int_{D} f(y) d y \quad \text { for }|x|>\rho,
$$

and it follows that $u_{t}$ is integrable over $\mathbf{R}^{3}$. Since $0<u_{t} / u \leq u_{t} / u_{0}$, we deduce in addition that $u_{t} / u$ is integrable over $\mathbf{R}^{3}$ and, hence, that $E$ is well defined as a Lebesgue integral and is nonnegative.

Next, we note that

$$
F=\int \frac{f}{u} d x=\int_{D} \frac{f}{u} d x \leq \frac{1}{u_{0}} \int_{D} f d x,
$$

and from this it is clear that $F$ too is well defined and nonnegative.

In order to show that $G$ is well defined we return to Eq. (2.3) and calculate the heat flux vector $q=-\operatorname{grad} u$, which proves to be

$\frac{1}{4 \pi} \int_{D}\left[\frac{1}{|x-y|^{3}} \operatorname{erfc}\left(\frac{|x-y|}{2 t^{1 / 2}}\right)+\frac{1}{|x-y|^{2}} \cdot \frac{1}{(\pi t)^{1 / 2}} \exp \left(-\frac{|x-y|^{2}}{4 t}\right)\right](x-y) f(y) d y$.

Thus, if we use Eq. (2.4) to estimate the complementary error function, we have

$$
|q| \leq \frac{1}{4 \pi^{3 / 2}} \int_{D}\left[\frac{2 t^{1 / 2}}{|x-y|^{3}}+\frac{1}{|x-y| t^{1 / 2}}\right] \exp \left(-\frac{|x-y|^{2}}{4 t}\right) f(y) d y
$$

and, hence, we have

$$
|q| \leq \frac{1}{4 \pi^{3 / 2}}\left[\frac{2 t^{1 / 2}}{(|x|-\rho)^{3}}+\frac{1}{(|x|-\rho) t^{1 / 2}}\right] \exp \left(-\frac{(|x|-\rho)^{2}}{4 t}\right) \int_{D} f(y) d y
$$

for $|x|>\rho$. This estimate shows that $|q|^{2}$ is integrable over $\mathbf{R}^{3}$ and, thus, that

$$
G=\int \frac{|q|^{2}}{u^{2}} d x \leq \frac{1}{u_{0}^{2}} \int|q|^{2} d x
$$

is well defined. Clearly $G$ is nonnegative. 
We turn to establishing Eqs. (2.5) and (2.6). Since $E, F$, and $G$ exist as Lebesgue integrals, the monotone convergence theorem ensures that

$$
\begin{aligned}
& E=\lim _{r \rightarrow \infty} \int_{B_{r}} \frac{u_{t}}{u} d x, \\
& F=\lim _{r \rightarrow \infty} \int_{B_{r}} \frac{f}{u} d x, \\
& G=\lim _{r \rightarrow \infty} \int_{B_{r}} \frac{|q|^{2}}{u^{2}} d x,
\end{aligned}
$$

where $B_{r}$ is the ball $|x|<r$. On the other hand, if we set $P=B_{r}$ throughout the identity (1.10), let $r \rightarrow \infty$, and remember that $H$ is defined as in Eq. (1.14), we deduce that

$$
H=\lim _{r \rightarrow \infty} \int_{\partial B_{r}} \frac{\nu \cdot q}{u} d S .
$$

However, the estimate (2.8) enables us to conclude that if $x \in \partial B_{r}$, that is, if $|x|=r$, then

$$
|q(x, t)|=O\left(\frac{1}{r} \exp \left(-\frac{(r-\rho)^{2}}{4 t}\right)\right) \quad \text { as } r \rightarrow \infty .
$$

Hence, as $r \rightarrow \infty$,

$$
\begin{aligned}
\left|\int_{\partial B_{r}} \frac{\nu \cdot q}{u} d S\right| & \leq \int_{\partial B_{r}} \frac{|q|}{u} d S \\
& \leq \frac{4 \pi r^{2}}{u_{0}} O\left(\frac{1}{r} \exp \left(-\frac{(r-\rho)^{2}}{4 t}\right)\right)=o(1),
\end{aligned}
$$

and this concludes the proof of both Eqs. (2.5) and (2.6).

Let us suppose that we are given an arbitrary positive $t$ and that we can vary the heat supply $f$ at will within the collection $\mathscr{C}$. We ask how large it is possible for $E(f, t), F(f, t)$, and $G(f, t)$ to be. When $\Omega=\mathbf{R}^{3}$, the answer to this question is that neither $E$ nor $G$ is bounded above on $\mathscr{C}$, but

$$
F(f, t) \leq 4 \pi \delta / \operatorname{erfc}\left(\frac{\delta}{2 t^{1 / 2}}\right) \text { for all } f \in \mathscr{C},
$$

where, it will be recalled, $\delta$ is the diameter of $D$.

Thus, by controlling the heat supply, we can make both the rate of increase of entropy at time $t$ and the rate of generation of entropy at time $t$ due to internal heat conduction as large as we like. By contrast, though, we cannot make the inward flux of entropy at time $t$ due to the heat supply as large as we like. We conclude that, whenever the rate of increase of entropy is large, the major contribution to that rate of increase arises not directly from the heat supply that we control, even though the heat supply may be large, but only indirectly by way of the mechanism of internal heat conduction.

The main thrust of these conclusions is in line with the corresponding conclusions in the static case [3, Theorem 1], and, indeed, as $t \rightarrow \infty$, the upper bound (2.9) 
tends to the limiting value $4 \pi \delta$, which is precisely the upper bound on $F$ that was found in [3].

In order to establish our assertions it is enough to verify that $E$ is not bounded above and that $F$ satisfies the inequality (2.9), for then it will follow from Eq. (2.5) that $G$ cannot be bounded above.

The proof that $E$ is not bounded above hinges upon the following estimate: for all $x$ with $|x|>\rho$ and for all $y \in D$,

$$
\exp \left(-\frac{|x-y|^{2}}{4 t}\right)>\frac{1}{2}\left(\frac{\pi}{t}\right)^{1 / 2}(|x|-\rho)^{2} \frac{1}{|x-y|} \operatorname{erfc}\left(\frac{|x-y|}{2 t^{1 / 2}}\right) .
$$

This is a straightforward consequence of the estimate (2.4) on the complementary error function, with $\mu=|x-y| /\left(2 t^{1 / 2}\right)$, for (2.4) tells us that

$$
\begin{aligned}
\exp \left(-\frac{|x-y|^{2}}{4 t}\right) & >\pi^{1 / 2} \frac{|x-y|}{2 t^{1 / 2}} \operatorname{erfc}\left(\frac{|x-y|}{2 t^{1 / 2}}\right) \\
& =\frac{1}{2}\left(\frac{\pi}{t}\right)^{1 / 2}|x-y|^{2} \cdot \frac{1}{|x-y|} \operatorname{erfc}\left(\frac{|x-y|}{2 t^{1 / 2}}\right),
\end{aligned}
$$

and, since $|x-y|>|x|-\rho$ if $|x|>\rho$ and $y \in D$, we see that (2.10) is correct.

If now we multiply both sides of (2.10) through by $f(y)$ and integrate with respect to $y$ over the domain $D$, we arrive at the inequaltiy

$$
\begin{aligned}
& \int_{D} \exp \left(-\frac{|x-y|^{2}}{4 t}\right) f(y) d y \\
& \quad \geq \frac{1}{2}\left(\frac{\pi}{t}\right)^{1 / 2}(|x|-\rho)^{2} \int_{D} \frac{1}{|x-y|} \operatorname{erfc}\left(\frac{|x-y|}{2 t^{1 / 2}}\right) f(y) d y \text { for }|x|>\rho .
\end{aligned}
$$

Let $n$ be any positive integer, and let $f$ be any heat supply that belongs to the collection $\mathscr{C}$ and that does not vanish identically. Then, by virtue of the cone property (1.5), $n f \in \mathscr{C}$; and if we calculate $E(n f, t)$ with the aid of Eqs. (1.11), (2.3), and (2.7), we see that

$$
E(n f, t)=\int I_{n} d x
$$

the integrand $I_{n}(x, t)$ being the ratio

$$
\frac{\frac{n}{(4 \pi t)^{3 / 2}} \int_{D} \exp \left(-\frac{|x-y|^{2}}{4 t}\right) f(y) d y}{u_{0}+\frac{n}{4 \pi} \int_{D} \frac{1}{|x-y|} \operatorname{erfc}\left(\frac{|x-y|}{2 t^{1 / 2}}\right) f(y) d y}
$$

For each $t>0,\left\{I_{n}\right\}$ is an increasing sequence of integrable functions of $x$ on $\mathbf{R}^{3}$ that converges to the limit

$$
\frac{\frac{1}{2 \pi^{1 / 2} t^{3 / 2}} \int_{D} \exp \left(-\frac{|x-y|^{2}}{4 t}\right) f(y) d y}{\int_{D} \frac{1}{|x-y|} \operatorname{erfc}\left(\frac{|x-y|}{2 t^{1 / 2}}\right) f(y) d y}
$$

as $n \rightarrow \infty$. If $E$ were bounded above on $\mathscr{C}$, we should conclude from the monotone convergence theorem that the limit $(2.12)$ must be an integrable function of $x$ on 
$\mathbf{R}^{3}$. However, the estimate (2.11) tells us that this cannot be so since $(|x|-\rho)^{2}$ is not an integrable function of $x$ for $|x|>\rho$. Thus $E$ cannot be bounded above on $\mathscr{C}$.

Finally, we prove Eq. (2.9) by noting that

$$
F=\int \frac{f}{u} d x=\int_{D} \frac{f}{u} d x
$$

In view of the definition (1.7) of the diameter $\delta$ and of the fact that $\mu^{-1} \operatorname{erfc}(\mu)$ is a decreasing function of $\mu$, it must be that

$$
\frac{1}{|x-y|} \operatorname{erfc}\left(\frac{|x-y|}{2 t^{1 / 2}}\right) \geq \frac{1}{\delta} \operatorname{erfc}\left(\frac{\delta}{2 t^{1 / 2}}\right) \quad \text { for } x, y \in D .
$$

It now follows from Eqs. (2.3) that

$$
u(x, t) \geq \frac{1}{4 \pi \delta} \operatorname{erfc}\left(\frac{\delta}{2 t^{1 / 2}}\right) \int_{D} f(y) d y \quad \text { for } x \in D,
$$

and when we combine this estimate with (2.13) we arrive at (2.9).

3. Behaviour of $E, F, G, H$ when $\Omega$ is a half-space. If the conductor occupies a domain $\Omega \neq \mathbf{R}^{3}$ and has a boundary that is maintained at constant temperature, then $H$ need no longer be identically zero. We illustrate this by considering what happens when $\Omega$ is the half-space $x_{1}>0$ and its boundary $\partial \Omega$ is the plane $x_{1}=0$. The advantage of this choice is that the solution to the appropriate initial-and-boundary value problem for the inhomogeneous heat equation can be constructed explicitly by the method of images [5, pp. 273-281]. In order to prove similar results for domains of more complicated geometry it would be necessary to devise arguments which do not depend upon having to find explicit solutions of initial-and-boundary value problems for the inhomogeneous heat equation.

We presume once again that we are given a nonempty, bounded domain $D \subset \Omega$ and we presume, in addition, that the distance from $D$ to $\partial \Omega$ is positive; that is, we presume there to be a constant $\varepsilon$ such that

$$
x_{1} \geq \varepsilon>0 \text { for all } x=\left(x_{1}, x_{2}, x_{3}\right) \in D .
$$

As before, $\mathscr{C}$ is the collection of all heat supplies $f$ that meet the conditions (1.2), (1.3), and (1.4), and, once again, $\mathscr{C}$ is a cone.

Now let $u_{0}$ be a positive constant. We shall require the temperature $u(x, t)$ to satisfy the differential equation (1.1), together with the initial condition

$$
u(x, 0)=u_{0} \text { for all } x \in \Omega,
$$

the condition

$$
u(x, t) \rightarrow u_{0} \quad \text { as }|x| \rightarrow \infty \quad \text { for } t>0,
$$

and the boundary condition

$$
u(x, t)=u_{0} \quad \text { for all } x \in \partial \Omega \text { and } t>0 .
$$


The solution of the initial-and-boundary value problem consisting of Eqs. (1.1), (3.2), (3.3), and (3.4) is

$$
u(x, t)= \begin{cases}u_{0}+\frac{1}{4 \pi} \int_{D}\left[\frac{1}{|x-y|} \operatorname{erfc}\left(\frac{|x-y|}{2 t^{1 / 2}}\right)\right. & \text { for } t>0, \\ \left.-\frac{1}{|\bar{x}-y|} \operatorname{erfc}\left(\frac{|\bar{x}-y|}{2 t^{1 / 2}}\right)\right] f(y) d y & \text { for } t=0,\end{cases}
$$

where $\bar{x}=\left(-x_{1}, x_{2}, x_{3}\right)$ is the image of the point $x=\left(x_{1}, x_{2}, x_{3}\right)$ under reflection in the plane $x_{1}=0$.

In much the same way as we proved the corresponding result of Sec. 2 we can prove that if $f \in \mathscr{C}$, Eq. (3.5) defines a function $u$ that is continuous for $t \geq 0$, that satisfies the initial condition (3.2) and the conditions (3.3) and (3.4), and that also satisfies the differential equation (1.1) for $t>0$.

For any pair of points $x, y \in \Omega$,

$$
|\bar{x}-y|^{2}-|x-y|^{2}=4 x_{1} y_{1}>0
$$

and, since $\mu^{-1} \operatorname{erfc}(\mu)$ decreases as $\mu$ increases, the explicit representation (3.5) confirms that $u(x, t) \geq u_{0}>0$ for $x \in \Omega$ and $t \geq 0$, as we should expect to be the case. This means, in particular, that it is legitimate to divide by $u$ whenever we have occasion to do so.

Next, we define $E, F, G$, and $H$ by way of Eqs. (1.11), (1.12), (1.13), and (1.14), respectively, the integrals being extended over the half-space $\Omega$. In fact: for each $f \in \mathscr{C}$ and each $t>0, E, F$, and $G$ exist as Lebesgue integrals and, hence, $H$ is well defined by Eq. (1.14); moreover, each of $E, F, G$, and $H$ is nonnegative.

The verification that $E, F$, and $G$ exist can be carried out along similar lines to those followed in Sec. 2. The fact that $E, F, G$, and $H$ are all nonnegative will emerge latter.

As we did in Sec. 2, we suppose that we are given an arbitrary positive $t$ and that we can vary the heat supply $f$ at will within the collection $\mathscr{C}$. We ask how large $E(f, t), F(f, t), G(f, t)$, and $H(f, t)$ can possibly be. When $\Omega$ is a half-space, the answer to this question is provided by the following statement, which constitutes the main result of this section: none of $E, G, H$ is bounded above on $\mathscr{C}$, but $F$ is bounded above on $\mathscr{C}$.

In order to prove that $E$ is not bounded above we need the following estimate: for all $x \in \Omega$ with $|x|>\rho$ and for all $y \in D$,

$$
\begin{aligned}
\exp ( & \left.-\frac{|x-y|^{2}}{4 t}\right)-\exp \left(-\frac{|\bar{x}-y|^{2}}{4 t}\right) \\
> & \frac{1}{2}\left(\frac{\pi}{t}\right)^{1 / 2}\left(1-\exp \left(-\frac{\varepsilon x_{1}}{t}\right)\right)(|x|-\rho)^{2} \\
& \cdot\left[\frac{1}{|x-y|} \operatorname{erfc}\left(\frac{|x-y|}{2 t^{1 / 2}}\right)-\frac{1}{|\bar{x}-y|} \operatorname{erfc}\left(\frac{|\bar{x}-y|}{2 t^{1 / 2}}\right)\right] .
\end{aligned}
$$


This estimate is a counterpart to estimate (2.10). In order to prove it we start from identity (3.6), which implies that

$$
\begin{aligned}
\exp & \left(-\frac{|x-y|^{2}}{4 t}\right)-\exp \left(-\frac{|\bar{x}-y|^{2}}{4 t}\right) \\
& =\left(1-\exp \left(-\frac{x_{1} y_{1}}{t}\right)\right) \exp \left(-\frac{|x-y|^{2}}{4 t}\right) \\
& >\left(1-\exp \left(-\frac{\varepsilon x_{1}}{t}\right)\right) \exp \left(-\frac{|x-y|^{2}}{4 t}\right) .
\end{aligned}
$$

On the other hand, the estimate (2.4) on the complementary error function, with $\mu=|x-y| /\left(2 t^{1 / 2}\right)$, tells us that

$$
\begin{aligned}
\exp \left(-\frac{|x-y|^{2}}{4 t}\right) & >\pi^{1 / 2} \frac{|x-y|}{2 t^{1 / 2}} \operatorname{erfc}\left(\frac{|x-y|}{2 t^{1 / 2}}\right) \\
& =\frac{1}{2}\left(\frac{\pi}{t}\right)^{1 / 2}|x-y|^{2} \cdot \frac{1}{|x-y|} \operatorname{erfc}\left(\frac{|x-y|}{2 t^{1 / 2}}\right) .
\end{aligned}
$$

Since $|x-y|>|x|-\rho$ if $|x|>\rho$ and $y \in D$, it must be that

$$
\exp \left(-\frac{|x-y|^{2}}{4 t}\right)>\frac{1}{2}\left(\frac{\pi}{t}\right)^{1 / 2}(|x|-\rho)^{2} \cdot \frac{1}{|x-y|} \operatorname{erfc}\left(\frac{|x-y|}{2 t^{1 / 2}}\right)
$$

and $a$ fortiori that

$$
\begin{aligned}
\exp \left(-\frac{|x-y|^{2}}{4 t}\right)> & \frac{1}{2}\left(\frac{\pi}{t}\right)^{1 / 2}(|x|-\rho)^{2} \\
& \cdot\left[\frac{1}{|x-y|} \operatorname{erfc}\left(\frac{|x-y|}{2 t^{1 / 2}}\right)-\frac{1}{|\bar{x}-y|} \operatorname{erfc}\left(\frac{|\bar{x}-y|}{2 t^{1 / 2}}\right)\right] .
\end{aligned}
$$

Combining (3.8) with (3.9) we arrive at (3.7).

If we multiply both sides of (3.7) through by $f(y)$ and integrate with respect to $y$ over the domain $D$, we see that

$$
\begin{aligned}
& \int_{D}\left[\exp \left(-\frac{|x-y|^{2}}{4 t}\right)-\exp \left(-\frac{|\bar{x}-y|^{2}}{4 t}\right)\right] f(y) d y \\
&> \frac{1}{2}\left(\frac{\pi}{t}\right)^{1 / 2}\left(1-\exp \left(-\frac{\varepsilon x_{1}}{t}\right)\right)(|x|-\rho)^{2} \\
& \quad \int_{D}\left[\frac{1}{|x-y|} \operatorname{erfc}\left(\frac{|x-y|}{2 t^{1 / 2}}\right)-\frac{1}{|\bar{x}-y|} \operatorname{erfc}\left(\frac{|\bar{x}-y|}{2 t^{1 / 2}}\right)\right] f(y) d y .
\end{aligned}
$$

We can also complete the proof that $E$ is not bounded above on $\mathscr{C}$ in much the same way as we proved the same assertion when $\Omega=\mathbf{R}^{3}$. To this end, let $n$ be any positive integer and let $f$ be any heat supply that belongs to the collection $\mathscr{C}$ but that does not vanish identically. Then $n f \in \mathscr{C}$, and if we calculate $E(n f, t)$, we see that

$$
E(n f, t)=\int I_{n} d x,
$$


the integrand $I_{n}(x, t)$ being the ratio

$$
\frac{\frac{n}{(4 \pi t)^{3 / 2}} \int_{D}\left[\exp \left(-\frac{|x-y|}{4 t}\right)-\exp \left(-\frac{|\bar{x}-y|^{2}}{4 t}\right)\right] f(y) d y}{u_{0}+\frac{n}{4 \pi} \int_{D}\left[\frac{1}{|x-y|} \operatorname{erfc}\left(\frac{|x-y|}{2 t^{1 / 2}}\right)-\frac{1}{|\bar{x}-y|} \operatorname{erfc}\left(\frac{|\bar{x}-y|}{2 t^{1 / 2}}\right)\right] f(y) d y} .
$$

For each $t>0,\left\{I_{n}\right\}$ is an increasing sequence of integrable functions of $x$ on $\Omega$ that converges to the limit

$$
\frac{\frac{1}{2 \pi^{1 / 2} t^{3 / 2}} \int_{D}\left[\exp \left(-\frac{|x-y|^{2}}{4 t}\right)-\exp \left(-\frac{|\bar{x}-y|^{2}}{4 t}\right)\right] f(y) d y}{\int_{D}\left[\frac{1}{|x-y|} \operatorname{erfc}\left(\frac{|x-y|}{2 t^{1 / 2}}\right)-\frac{1}{|\bar{x}-y|} \operatorname{erfc}\left(\frac{|\bar{x}-y|}{2 t^{1 / 2}}\right)\right] f(y) d y}
$$

as $n \rightarrow \infty$. If $E$ were bounded above on $\mathscr{C}$, we would conclude from the monotone convergence theorem that the limit (3.13) must be an integrable function of $x$ on $\mathbf{R}^{3}$. However, the estimate (3.10) tells us that this cannot be the case since (1$\left.\exp \left(-\varepsilon x_{1} / t\right)\right)(|x|-\rho)^{2}$ is not an integrable function of $x$ for $|x|>\rho$ and $x_{1}>0$. Thus $E$ cannot be bounded above on $\mathscr{C}$.

We remark in passing that, on setting $n=1$ in (3.11) and (3.12), it becomes clear that $E$ is nonnegative.

We turn next to showing that $H$ cannot be bounded above. Since $E, F$, and $G$ exist as Lebesgue integrals, the monotone convergence theorem ensures that

$$
\begin{aligned}
& E=\lim _{r \rightarrow \infty} \int_{B_{r} \cap \Omega} \frac{u_{t}}{u} d x, \\
& F=\lim _{r \rightarrow \infty} \int_{B_{r} \cap \Omega} \frac{f}{u} d x, \\
& G=\lim _{r \rightarrow \infty} \int_{B_{r} \cap \Omega} \frac{|q|^{2}}{u^{2}} d x,
\end{aligned}
$$

the integrals being taken over the intersection of the ball $B_{r}$ with the half-space $\Omega$. Thus, if we set $P=B_{r} \cap \Omega$ throughout the identity (1.10), let $r \rightarrow \infty$, and remember that $H$ is defined as in Eq. (1.14), we deduce that

$$
H=\lim _{r \rightarrow \infty} \int_{\partial\left(B_{r} \cap \Omega\right)} \frac{\nu \cdot q}{u} d S .
$$

The boundary $\partial\left(B_{r} \cap \Omega\right)$ can be expressed as the union of the plane base $\Pi_{r}=$ $\left\{x: x_{1}=0\right.$ and $\left.|x|<r\right\}$ with the curved surface $\Sigma_{r}=\left\{x: x_{1} \geq 0\right.$ and $\left.|x|=r\right\}$, and so

$$
H=\lim _{r \rightarrow \infty}\left(\int_{\Pi_{r}}+\int_{\Sigma_{r}}\right) \frac{\nu \cdot q}{u} d S .
$$

By calculating the heat flux $q=-\operatorname{grad} u$ directly from the explicit solution (3.5) and making appropriate estimates on the decay of $q$ as $|x| \rightarrow \infty$, it is not difficult to show that

$$
\lim _{r \rightarrow \infty} \int_{\Sigma_{r}} \frac{\nu \cdot q}{u} d S=0
$$


and, hence, that

$$
H=\lim _{r \rightarrow \infty} \int_{\Pi_{r}} \frac{\nu \cdot q}{u} d S .
$$

Thus, in view of the boundary condition (3.4),

$$
H=\frac{1}{u_{0}} \lim _{r \rightarrow \infty} \int_{\Pi_{r}} \nu \cdot q d S .
$$

It is clear from Eq. (3.14) that $H$ has the property that

$$
H(n f, t)=n H(f, t)
$$

for every positive integer $n$ and every $f \in \mathscr{C}$ and so $H$ cannot be bounded above provided that there exists some $f \in \mathscr{C}$ for which $H(f, t)>0$. In order to show that there is such an $f$ and, in fact, that any $f \in \mathscr{C}$ that does not vanish identically has this property, we return to the differential equation (1.1), integrate both sides with respect to $x$ over the set $B_{r} \cap \Omega$, and appeal to the divergence theorem to deduce that

$$
\int_{\partial\left(B_{r} \cap \Omega\right)} \nu \cdot q d S=\int_{B_{r} \cap \Omega} f d x-\int_{B_{r} \cap \Omega} u_{t} d x,
$$

where

$$
\int_{\partial\left(B_{r} \cap \Omega\right)} \nu \cdot q d S=\left(\int_{\Pi_{r}}+\int_{\Sigma_{r}}\right) \nu \cdot q d S .
$$

Once again it is not difficult to show that

$$
\lim _{r \rightarrow \infty} \int_{\Sigma_{r}} \nu \cdot q d S=0
$$

so, on letting $r \rightarrow \infty$ in Eq. (3.15) we deduce the equation

$$
\lim _{r \rightarrow \infty} \int_{\Pi_{r}} \nu \cdot q d S=\int_{\Omega} f d x-\int_{\Omega} u_{t} d x=\int_{D} f d x-\int_{\Omega} u_{t} d x
$$

and when we combine this with (3.14) we see that

$$
H=\frac{1}{u_{0}}\left(\int_{D} f d x-\int_{\Omega} u_{t} d x\right) .
$$

Next we calculate the derivative $u_{t}$ from Eq. (3.5) and find that it can be expressed as the difference

$$
u_{t}(x, t)=\phi(x, t)-\phi(\bar{x}, t) \text { for } x \in \Omega \text { and } t>0,
$$

where

$$
\phi(x, t)=\frac{1}{(4 \pi t)^{3 / 2}} \int_{D} \exp \left(-\frac{|x-y|^{2}}{4 t}\right) f(y) d y .
$$

It is convenient to regard $\phi$ as being defined by Eq. (3.18) at all points $x \in \mathbf{R}^{3}$. Then, in view of Fubini's Theorem and the identity

$$
\int_{\mathbf{R}^{3}} \frac{1}{(4 \pi t)^{3 / 2}} \exp \left(-\frac{|x-y|^{2}}{4 t}\right) d x=1,
$$


we deduce from Eq. (3.18) that

$$
\int_{\mathbf{R}^{3}} \phi d x=\int_{D} f d x
$$

Thus, if we substitute from Eqs. (3.17) and (3.19) into Eq. (3.16), we see that

$$
\begin{aligned}
H & =\frac{1}{u_{0}}\left(\int_{\mathbf{R}^{3}} \phi(x, t) d x-\int_{\Omega} \phi(x, t) d x+\int_{\Omega} \phi(\bar{x}, t) d x\right) \\
& =\frac{1}{u_{0}}\left(\int_{\mathbf{R}^{3} \backslash \Omega} \phi(x, t) d x+\int_{\Omega} \phi(\bar{x}, t) d x\right) \\
& =\frac{2}{u_{0}} \int_{\mathbf{R}^{3} \backslash \Omega} \phi(x, t) d x .
\end{aligned}
$$

In summary, we have arrived at the expression

$$
H=\frac{1}{4 u_{0}(\pi t)^{3 / 2}} \int_{\mathbf{R}^{3} \backslash \Omega}\left(\int_{D} \exp \left(-\frac{|x-y|^{2}}{4 t}\right) f(y) d y\right) d x,
$$

which is clearly positive if $f \in \mathscr{C}$ and $f$ does not vanish identically. As we have seen, it follows that $H$ cannot be bounded above on $\mathscr{C}$.

In order to deal with $F$ we note that

$$
F=\int \frac{f}{u} d x=\int_{D} \frac{f}{u} d x
$$

from which equation it is clear that $F$ is nonnegative. If we put

$$
m(t)=\inf \left\{\frac{1}{|x-y|} \operatorname{erfc}\left(\frac{|x-y|}{2 t^{1 / 2}}\right)-\frac{1}{|\bar{x}-y|} \operatorname{erfc}\left(\frac{|\bar{x}-y|}{2 t^{1 / 2}}\right): x, y \in D\right\},
$$

then $m(t)>0$ and we deduce from Eq. (3.5) that

$$
u(x, t) \geq \frac{m(t)}{4 \pi} \int_{D} f(y) d y \quad \text { for } x \in D \text { and } t>0,
$$

and, therefore, that

$$
F=\int_{D} \frac{f}{u} d x \leq \frac{4 \pi}{m(t)} \text { for all } f \in \mathscr{C}
$$

Thus $F$ is bounded above on $\mathscr{C}$.

Finally, we note that $G$, as defined by Eq. (1.13), is clearly nonnegative, and since $E+H=G+F$, where $E$ and $H$ are not bounded above while $F$ is bounded above, it must be that $G$ is not bounded above.

\section{REFERENCES}

[1] C. Truesdell, The Tragicomical History of Thermodynamics, , 1822-1854, Springer-Verlag, New York, 1980

[2] W. A. Day, Parabolic equations and thermodynamics, Quart. Appl. Math. 50, 523-533 (1992)

[3] W. A. Day, Entropy and elliptic equations, Quart. Appl. Math. 51, 191-200 (1993)

[4] G. Hellwig, Partial Differential Equations, Blaisdell, New York, 1964

[5] H. S. Carslaw and J. C. Jaeger, Conduction of Heat in Solids, 2nd edition, Clarendon Press, Oxford, 1959 